\title{
Clinical Observations Date Time of Reference Timepoint
}

National Cancer Institute

\section{Source}

National Cancer Institute. Clinical Observations Date Time of Reference Timepoint. NCI

Thesaurus. Code C119808.

The date and time of a specific reference point for the clinical observation. 